\section{AVALIAÇÃO DA FUNÇÃO SEXUAL EM UM GRUPO DE MASTECTOMIZADAS}

\author{
Evaluation of sexual function in a group of mastectomized \\ women
}

Evaluación dela función sexualen un grupodemastectomizadas
Artigo Original

Izabelle Quintiliano Montenegro Bomfim $^{(1)}$

Rosana Priscila Santos Batista ${ }^{(1)}$ Renata Melo de Carvalho Lima ${ }^{(1)}$

socioeconômica, estado civil e escolaridade) e ginecológicos (paridade, tempo pós-cirúrgico e tipo de cirurgia), além do Questionário Quociente Sexual (QS-F) - versão feminina. Os resultados foram analisados através da estatística descritiva e dos testes Kruskal-Wallis e Spearman, considerando-se $\mathrm{p} \leq 0,05$. Resultados: Encontrou-se um desempenho sexual de nulo a ruim em $35,3 \%(n=12)$ das mulheres, e apenas $11,8 \%(n=4)$ apresentaram um escore favorável no QS-F. Observou-se uma maior porcentagem de mulheres apresentando dificuldade na excitação com $88,2 \%(n=30)$, e quanto ao estado civil e escolaridade, $47,1 \%$ $(n=16)$ eram casadas e 55,9\% $(n=19)$ apresentaram escolaridade até ensino fundamental. Conclusão: As mulheres mastectomizadas investigadas apresentaram função sexual baixa e insatisfatória de acordo com o QS-F, eram casadas e apresentavam até o ensino fundamental.

Descritores: Mastectomia; Sexualidade; Saúde da Mulher.

\title{
ABSTRACT
}

Objective: To evaluate the sexual function of mastectomized women. Methods: crosssectional descriptive study conducted between September and October 2012 with 34 mastectomized women, members of a support group, who were sexually active in the last six months. It was used an instrument for collecting sociodemographic (socioeconomic status, marital status and education) and gynecological data (parity, post-surgical time and type of surgery) in addition to the Female Sexual Quotient (FSQ) questionnaire. Results were analyzed using descriptive statistics, the Kruskal-Wallis and Spearman's test with $p \leq 0.05$. Results: It was verified a sexual performance that ranged from null to bad in $35.3 \%(n=12)$ of women, while only $11.8 \%(n=4)$ had a favorable score on FSQ. It was observed a higher percentage of women presenting difficulty in sexual arousal, $88.2 \%(n=30)$. Regarding marital status and education, $47.1 \%(n=16)$ were married and $55.9 \%(n=19)$ had studied up to elementary school. Conclusion: The investigated mastectomized women presented low and unsatisfactory sexual function according to the FSQ; they were married and studied up to elementary school.

Descriptors: Mastectomy; Sexuality; Women's Health.

\section{RESUMEN}

Objetivo: Evaluar la función sexual de mujeres mastectomizadas. Métodos: Estudio transversal y descriptivo realizado entre septiembre y octubre de 2012, con 34 mujeres mastectomizadas, sexualmente activas en los últimos 6 meses y participantes en un grupo de apoyo. Se utilizó un instrumento para recogida de datos sociodemográficos (condición socioeconómica, estado civil y escolaridad) y ginecológicos (paridad, tiempo postquirúrgico y tipo de cirugía) además del Cuestionario Cociente Sexual (CS-F) - versión femenina. Los resultados fueron analizados a través de la estadística descriptiva y las pruebas de Kruskal1) Universidade Estadual de Ciências da
Saúde de Alagoas - UNCISAL - Maceió
(AL) - Brasil

Recebido em: 05/03/2013 Revisado em: 20/07/2013 Aceito em: 06/12/2013 
Wallis y Spearman, considerando $p \leq 0,05$. Resultados: Se encontró un rendimiento sexual de nulo a malo en el 35,3\% $(n=12)$ de las mujeres y solamente el 11,8\% $(n=4)$ presentaron una puntuación favorable del CS-F. Se observó mayor porcentaje de mujeres con dificultad de excitación en el $88,2 \%(n=30)$, y respecto al estado civil y escolaridad, el 47,1\% $(n=16)$ eran casadas y el $55,9 \%(n=19)$ presentaron escolaridad máxima de educación básica. Conclusión: Las mujeres mastectomizadas investigadas presentaron baja e insatisfactoria función sexual según el CS-F, eran casadas y tenían escolaridad máxima de educación básica.

Descriptores: Mastectomía; Sexualidad; Salud de la Mujer.

\section{INTRODUÇÃO}

No Brasil, dentre as neoplasias malignas, o câncer de mama é o tipo mais frequente, o mais comum entre as mulheres ${ }^{(1,2)}$. O Instituto Nacional de Câncer (INCA) estimou 57.120 novos casos para o ano de 2014, e no ano de 2008, o número de mortes foi 12.098, sendo 11.969 mulheres $^{(1,2)}$.

Pelo fato de ser uma doença cujo diagnóstico ainda é tardio, ocasionando estágios avançados, o sucesso do tratamento diminui, fazendo com que as taxas de mortalidade do câncer de mama continuem elevadas, apesar do bom prognóstico quando diagnosticado e tratado precocemente ${ }^{(1)}$.

Uma das formas de tratamento para o câncer de mama é a cirurgia, a qual pode ser conservadora e não conservadora. As cirurgias conservadoras, caracterizadas pela retirada de um segmento da mama, consistem na tumorectomia e quadrantectomia ${ }^{(3)}$. Já as cirurgias não conservadoras, chamadas de mastectomias, consistem na retirada da mama e podem ser divididas em: mastectomia radical modificada, encontrada em duas versões (tipo Patey e tipo Madden); mastectomia radical de Halsted; e a mastectomia total ${ }^{(4)}$. A mastectomia, pelo seu caráter agressivo e traumatizante para a vida e saúde da mulher, proporciona alterações na sua imagem corporal, identidade e autoestima, podendo repercutir na sua qualidade de vida e, consequentemente, em sua sexualidade, mesmo nas pacientes que tiveram uma vida sexual satisfatória antes da doença ${ }^{(5)}$.

Os efeitos adversos do tratamento, principalmente da quimioterapia, levam a quadros de atrofia vulvovaginal, dispareunia, irritação e secura vaginal, inibição do desejo e da excitação, anorgasmia, menopausa prematura, náuseas, vômitos, fadiga e alopecia, o que pode impedir o funcionamento sexual satisfatório. Apesar de os efeitos fisiológicos tenderem a diminuir com o tempo, o dano à função sexual pode persistir por anos em sobreviventes de vários tipos de câncer ${ }^{(5,6)}$.

As disfunções sexuais se caracterizam por falta de desejo, ausência de orgasmo, disfunção na excitação, desconforto e/ou dor no ato sexual, o que prejudica uma ou mais das fases desse ciclo (desejo, excitação, orgasmo e resolução), podendo inclusive bloqueá-lo em determinado momento do seu desenrolar ${ }^{(7)}$. Seus principais fatores de risco podem ser divididos em físicos, psicológicos, psicossociais e sociodemográficos ${ }^{(8,9)}$.

Uma forma de avaliar essas disfunções é através da aplicação de questionários e, dentre os específicos em sexualidade, destaca-se o Questionário Quociente Sexual - versão feminina (QS-F), que avalia a função sexual em mulheres sexualmente ativas, elaborado para a população brasileira. Pode também ser utilizado para a estratificação de pacientes em estudos clínicos ou observacionais, bem como para a mensuração da eficácia de intervenção que objetiva o tratamento das disfunções sexuais da mulher ${ }^{(8)}$.

Sabendo que o quadro de disfunção sexual é reconhecido pela Organização Mundial de Saúde como um problema de saúde pública ${ }^{(10)}$, considera-se de suma importância abordar a sexualidade humana à mulher mastectomizada que sofreu danos e lesões não somente físicas como também emocionais, repercutindo em sua qualidade de vida, visto que ela deve ter atenção integral de saúde pelos profissionais que lidam com a saúde pública ${ }^{(11)}$.

Portanto, esta pesquisa teve como objetivo avaliar a função sexual de mulheres mastectomizadas.

\section{MÉTODOS}

Estudo transversal descritivo, cuja coleta de dados ocorreu nos meses de setembro e outubro de 2012, com as participantes do Grupo de Mama Renascer - Organização Não Governamental (ONG) GRUMARE, localizada no Centro Especializado em Reabilitação Municipal (PAM) Salgadinho, da cidade de Maceió-AL, que apresentavam prontuários no banco de dados da ONG.

O trabalho foi composto por uma amostra censitária com todas ( $\mathrm{n}=63)$ as mulheres que participavam da ONG no período da coleta. Elegeram-se como critérios para inclusão na pesquisa as mulheres mastectomizadas participantes do Grupo de Mama Renascer e ativas sexualmente nos últimos 6 meses. Excluíram-se as mulheres que estavam em vigência de tratamento quimioterápico ou radioterápico para o câncer de mama, mulheres que realizaram técnicas conservadoras como tratamento, mulheres que apresentaram recidiva da doença e as que não foram encontradas no período da coleta. Assim, das 63 mulheres participantes da ONG, 9 não apresentaram relação sexual nos últimos 6 meses, 12 não 
foram encontradas, 4 estavam em vigência de tratamento quimioterápico ou radioterápico e 4 se recusaram a participar do estudo, perfazendo um total de 29 exclusões. A amostra constou, então, de 34 mulheres após os critérios de inclusão e exclusão.

Após a coleta dos prontuários, contataram-se as mulheres de duas maneiras: através da presença na reunião mensal que ocorre toda a primeira quarta-feira do mês na própria sede da ONG ou através de visita domiciliar.

Realizou-se a aplicação dos instrumentos de coleta de dados em apenas um encontro, no qual se explicou os objetivos da pesquisa e, após demonstrarem o interesse em participar, as mulheres investigadas assinaram o Termo de Consentimento Livre e Esclarecido.

$\mathrm{O}$ primeiro instrumento aplicado pelas pesquisadoras abordava os seguintes itens: idade, nível sociodemográfico e escolaridade. Para a caracterização de dados ginecológicos e obstétricos, coletaram-se as variáveis "paridade", "tempo pós-cirúrgico" e "tipo de cirurgia".

O tipo de cirurgia dependeu do estadiamento clínico e do tipo histológico de cada mulher, analisando a característica do tumor e a fase em que a doença era diagnosticada, podendo ser classificada como cirurgia conservadora (tumorectomia e quadrantectomia ${ }^{(12)}$ e não conservadora (mastectomias, mastectomia radical modificada, mastectomia radical de Halsted $^{(12-14)}$. A mastectomia radical modificada pode ser encontrada em duas versões: tipo Patey, em que o músculo peitoral menor, a glândula mamária, III, IV e V espaços intercostais não são conservados e ocorre o esvaziamento radical axilar; e tipo Madden, em que há a preservação dos músculos peitoral maior e menor e dos espaços intercostais. Nesta variável de mastectomia, não existe o esvaziamento axilar, tendo indicação os casos de carcinoma ductal in $\operatorname{situ}^{(12,13)}$. Já na mastectomia radical de Halsted, a mama, a papila e os músculos peitorais (maior e menor) subjacentes não são preservados, incluindo a linfadenectomia axilar, muitas vezes necessitando da realização de enxerto ${ }^{(13,14)}$.

Para definir o nível sociodemográfico, a presente pesquisa baseou-se no Critério de Classificação Econômica Brasil $2008^{(15)}$, que utiliza características domiciliares para atribuir pontos e realiza a soma destes para definir a classe econômica, podendo ser A1, A2, B1, B2, C1, C2, D, E, sendo $\mathrm{A} 1$ atribuído à maior somatória (42-46) e $\mathrm{E}$, à menor $(0-7)^{(15)}$.

O formulário sociodemográfico e o Questionário Quociente Sexual - versão feminina (QS-F) foram aplicados pelos pesquisadores em local reservado, em uma única entrevista, com duração média de 20 minutos. O QS-F ${ }^{(9)}$ avalia o desempenho da função sexual nas mulheres e o domínio por domínio dessa atividade, pela consideração individualizada das questões pertinentes aos diferentes aspectos investigados. Esse questionário é validado ${ }^{(9)}$, sendo composto por 10 perguntas, arranjadas em cinco domínios (desejo e interesse sexual, preliminares, excitação da mulher e sintonia com o parceiro, conforto na relação sexual e orgasmo e satisfação sexual), com escores que são: nunca $(0)$, raramente (1), às vezes (2), aproximadamente $50 \%$ das vezes (3), a maioria das vezes (4) e sempre (5). Para obter o resultado é necessário somar os pontos atribuídos a cada questão, subtrair 5 pontos da questão 7 e multiplicar o total por 2: 2 x (Q1 + Q 2 + Q 3 + Q $4+\mathrm{Q} 5+\mathrm{Q} 6+[5-\mathrm{Q} 7]+\mathrm{Q}$ $8+$ Q9 + Q 10) (Q = questão $[5-\mathrm{Q} 7]=$ a questão 7 requer que se faça previamente essa subtração e que o resultado entre na soma das questões ${ }^{(9)}$.

O resultado do quociente é de quanto maior o escore, melhor o padrão de desempenho sexual: de 82 a 100 pontos, bom a excelente; de 62 a 80 pontos, regular a bom; de 42 a 60 pontos, desfavorável a regular; de 22 a 40 pontos, ruim a desfavorável; de 0 a 20 pontos, nulo a ruim. Estabeleceu-se durante a sua validação o ponto de corte em 60 como forma de rastreamento para a disfunção sexual feminina ${ }^{(9)}$.

Em seguida à aplicação dos instrumentos, convidaramse as mulheres investigadas a participarem de uma palestra na própria $\mathrm{ONG}$, em uma data previamente marcada.

Após o período de avaliações, os dados encontrados foram tratados através de estatística descritiva, demonstrados em tabelas e gráficos. Para a determinação da associação entre a função sexual e as variáveis secundárias (tipo de mastectomia, idade, nível sociodemográfico, estado civil e tempo pós-cirúrgico), utilizou-se o teste não paramétrico de Kruskal-Wallis e o coeficiente de correlação de Spearman, considerando-se o nível de significância de $\mathrm{p} \leq 0,05$, por meio do programa SPSS versão 17.0.

A presente pesquisa recebeu a aprovação do Comitê de Ética da Universidade Estadual de Ciências da Saúde de Alagoas (UNCISAL), com o protocolo de número 1870, e obedeceu aos princípios éticos dispostos na Resolução $\mathrm{n}^{\circ}$ 196/96 do Conselho Nacional de Saúde, do Ministério da Saúde (CNS/MS).

\section{RESULTADOS}

Encontrou-se a média de idade das 34 mulheres participantes da pesquisa de $52,3( \pm 11,1)$ anos, sendo 25 a idade mínima e 72 a idade máxima. A Tabela I descreve as características da amostra quanto ao aspecto sociodemográfico (condição socioeconômica, estado civil e escolaridade). No que diz respeito ao estado civil e escolaridade, $47,1 \%(n=16)$ eram casadas e $55,9 \%(n=19)$ apresentavam escolaridade até o ensino fundamental.

Observou-se na amostra estudada um predomínio da classe $\mathrm{C}$, com $50 \%(\mathrm{n}=17)$ das mulheres. Outro ponto 
analisado indicou que quanto maior a classe social, maior a sua pontuação no QS-F, em que a classe B apresenta a média de pontuação 50,6 (desfavorável a regular) e a classe $\mathrm{D}(31,7)$ e $\mathrm{C}(22,5)$ variam de ruim a desfavorável $(\mathrm{r}=0,349$; $\mathrm{p}=0,043)$. A comparação entre os valores da pontuação no QS-F apresentou diferença significativa entre a distribuição das médias por classe social $(\mathrm{p}=0,012)$.

Com relação aos antecedentes gineco-obstétricos das mulheres mastectomizadas entrevistadas, pode-se observar, através da Tabela II, a paridade de destaque, com $67,7 \%$ $(n=23)$ nas que tiveram 2 filhos ou menos e $50 \%(n=17)$ das mulheres relataram ter realizado a mastectomia há mais de 5 anos. Quanto ao tipo de cirurgia, $41,2 \%(n=14)$ realizaram a mastectomia total, seguidas pela mastectomia radical de Halsted com 26,5\% ( $\mathrm{n}=9)$.
Das 34 mulheres entrevistadas, a função sexual encontrada através do QS-F indicou desempenho sexual nulo a ruim em 35,3\% ( $\mathrm{n}=12)$ delas (Figura 1). De acordo com os aspectos abordados no QS-F e a distribuição das respostas das mulheres, pode-se observar, na Figura 2, uma maior porcentagem de mulheres apresentando dificuldade na excitação com $88,2 \% \quad(n=30)$, seguida do domínio "desejo e interesse sexual", com 82,4\% (n=28) delas com desejo ou interesse sexual hipoativo.

Através dos domínios do QS-F, as mulheres que tinham realizado a mastectomia total representaram as que obtiveram a menor média de pontuação - 15,9 (nulo a ruim) -, sendo a melhor resposta ao questionário as que realizaram a mastectomia radical modificada do tipo Madden, com média de pontuação de 62,4 (regular a bom)

Tabela I - Caracterização sociodemográfica da amostra das mulheres mastectomizadas pertencentes à ONG GRUMARE. Maceió-AL, 2012.

\begin{tabular}{llcc}
\hline Variáveis & Categoria & $\mathbf{n}$ & $\mathbf{\%}$ \\
\hline Nível socioeconômico & $\mathrm{B}$ & 10 & 29,4 \\
& $\mathrm{C}$ & 17 & 50,0 \\
\multirow{2}{*}{ Estado civil } & $\mathrm{D}$ & 7 & 20,6 \\
& Casada & 16 & 47,1 \\
& Separada/divorciada & 3 & 8,8 \\
& Solteira & 11 & 32,4 \\
Escolaridade & Viúva & 4 & 11,8 \\
& Fundamental & 19 & 55,9 \\
& Ensino Médio & 8 & 23,5 \\
& Ensino Superior & 7 & 20,6 \\
\hline
\end{tabular}

$\mathrm{n}=$ número de indivíduos; $\%=$ porcentagem

Tabela II - Caracterização de dados ginecológicos e obstétricos da amostra total das mulheres mastectomizadas entrevistadas pertencentes à ONG GRUMARE. Maceió-AL, 2012.

\begin{tabular}{llcc}
\hline Variável & Categoria & n & \% \\
\hline Paridade & Até 2 filhos & 23 & 67,7 \\
& 3 filhos & 8 & 23,5 \\
\multirow{2}{*}{ Tempo pós-cirúrgico } & Mais de 3 filhos & 3 & 8,8 \\
& Menos de 1 ano & 6 & 17,6 \\
& 1 a 5 anos & 11 & 32,4 \\
Tipo de cirurgia & Mais de 5 anos & 17 & 50,0 \\
& Total & 14 & 41,2 \\
& Halsted & 9 & 26,5 \\
& Outras* & 11 & 32,4 \\
\hline
\end{tabular}

$\mathrm{n}=$ número de indivíduos; \% = porcentagem ; *Patten, Madden e não soube especificar 
$(\mathrm{p}=0,004)$. Esse valor de $\mathrm{p}$ é correspondente à comparação entre as distribuições das médias. Como o tipo de cirurgia não é uma variável numérica, não pode ser feita correlação com a pontuação do QS-F.

Quanto à idade das pacientes, verificou-se a existência de uma fraca correlação negativa com a pontuação do QS-F, indicando que quanto maior a idade, menor o escore nesse questionário $(\mathrm{r}=-0,360 ; \mathrm{p}=0,036)$. Não existiu diferença significativa entre tempo de cirurgia e pontuação no QS-F $(r=-0,303 ; p=0,198)$.

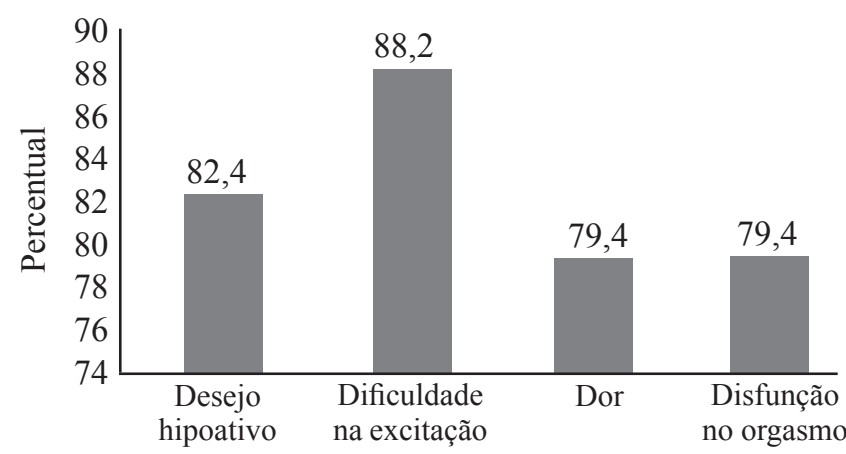

Figura 1 - Escore final do Quociente Sexual - versão feminina - das mulheres mastectomizadas participantes da pesquisa. Maceió-AL, 2012.

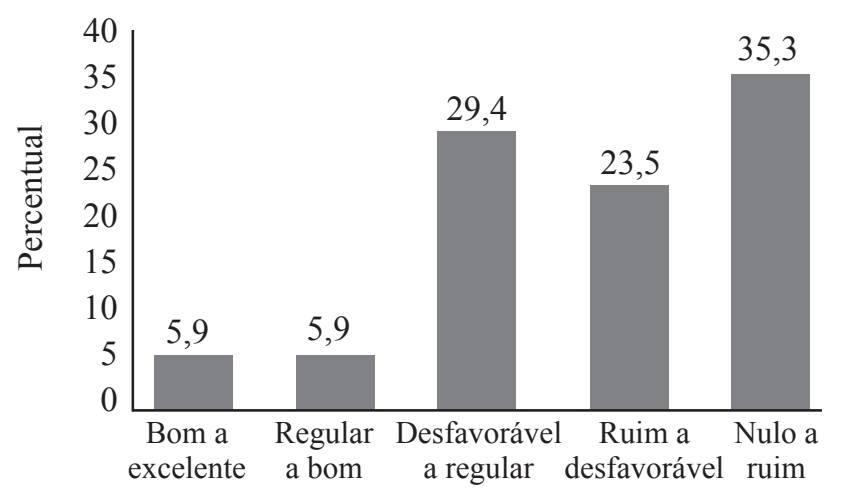

Figura 2 - Principais disfunções sexuais encontradas na amostra estudada. Maceió-AL, 2012.

\section{DISCUSSÃO}

Estudos sobre a sexualidade feminina e o câncer de mama vêm sendo conduzidos há anos, grande parte deles abordando o impacto psicológico e a qualidade de vida na saúde da mulher ${ }^{(2,4,16)}$. Outros estudos confrontam as técnicas cirúrgicas, ou seja, os benefícios das cirurgias conservadoras (tumorectomia, quadrantectomia) frente às mastectomias e à reconstrução mamária ${ }^{(17,18)} \mathrm{e}$ as consequências na qualidade de vida da mulher ${ }^{(4,19)}$.
Nesta presente pesquisa, a idade média das mulheres foi de 52,3 anos, corroborando os achados de outros estudos $^{(17,20,21)}$, nos quais a idade para diagnóstico variou de 45 a 60 anos, descendo com o passar do tempo e chegando a 35 anos $^{(1)}$. A partir de 2008, dados indicaram que o câncer de mama tem incidência mais alta em mulheres acima de 35 anos, e essa doença tende a crescer e assumir características progressivas a partir dessa idade ${ }^{(1)}$.

Pesquisas mostram que mulheres mastectomizadas mais jovens são acometidas com mais problemas em relação a sua sexualidade ${ }^{(4,22)}$, indicando que elas têm maior risco para estresse psicológico e disfunção sexual e maior dificuldade em se adaptar à nova imagem corporal, isso provavelmente pelo fato de terem menos preparo para enfrentar a situação, além de terem planos de maternidade adiados ou abandonados ${ }^{(4)}$. Isso não corrobora com a pesquisa em questão, na qual se observou que, quando a idade aumenta, ocorre uma diminuição na pontuação do QS-F.

No presente estudo, $47,1 \%$ das entrevistadas casadas apresentaram a categoria "desfavorável a regular" como resultado do QS-F. Já nas solteiras, observou-se maior predominância do escore "nulo a ruim". Em outro estudo(4), encontrou-se que as mulheres com um relacionamento estável tiveram resultados melhores quando comparadas às sem relacionamento estável.

Mais da metade $(52,9 \%)$ das mulheres entrevistadas estudaram até o nível fundamental, sendo essas as que obtiveram o pior escore no QS-F. Pode-se observar o mesmo resultado em outra pesquisa ${ }^{(20)}$, na qual foi possível analisar que $57 \%$ das mulheres apresentava baixa escolaridade, encontrando uma correlação estatisticamente significativa nesse aspecto. Em um estudo ${ }^{(17)}$, a maioria completou somente o $1^{\circ}$ grau, condizendo com o resultado encontrado na presente investigação. Também se percebeu em outro estudo(4) que a escolaridade pode afetar a vida sexual de mulheres mastectomizadas e as mulheres de nível universitário obtiveram escores melhores quando comparadas às que cursaram até o ensino médio.

Encontrou-se, na presente pesquisa, uma baixa condição socioeconômica, com $70,6 \%$ das mulheres entre classes C e D, justificado pelo fato de a ONG ser localizada em um centro de saúde e de atendimento médico da rede pública, e por terem dificuldade de acesso ao atendimento privado.

Um estudo ${ }^{(22)}$ mostrou que as mulheres de nível socioeconômico A e B obtiveram a melhor média dos escores referentes à sexualidade em comparação com as das classes $\mathrm{C}$ e $\mathrm{D} / \mathrm{E}$, corroborando com os achados da pesquisa em questão, quanto maior a classe social, maior foi a pontuação no QS-F com uma melhor função sexual. Metade 
das mulheres colaboradoras da atual pesquisa realizou a cirurgia há mais de 5 anos e não foi observada diferença significativa entre o tempo pós-cirúrgico e a pontuação do questionário, apesar de ter sido observada uma diminuição no escore com o passar do tempo de cirurgia. Outra pesquisa $^{(4)}$ mostra que o tempo decorrido desde a cirurgia não influencia nos aspectos de sexualidade. Já outro estudo $^{(23)}$, acompanhando 191 mulheres em tratamento para o câncer de mama, observou um aumento de problemas relacionados ao interesse e à atividade sexual com o passar do tempo, justificado pelas alterações corporais (alopécia, linfedema, cicatriz e amputação da mama) que ainda não foram assimiladas pela mulher, podendo causar prejuízo na sua autoimagem, consequentemente afetando sua vida sexual.

Todos os tipos de tratamento para o câncer de mama têm um impacto significativo na autoimagem, resultando em problemas sexuais ${ }^{(24,25)}$, entretanto, outros autores ${ }^{(23,26)}$ não encontraram efeitos significativos nos problemas sexuais na comparação entre os tipos de cirurgia, relatando que o único achado mais consistente é de que as mulheres que sofreram qualquer tipo de mastectomia, em vez da cirurgia conservadora, têm mais problemas com a imagem corporal.

No presente trabalho, as pacientes que fizeram mastectomia total obtiveram menor escore médio no QS-F. Em seguida, vieram as que se submeteram a mastectomia radical de Halsted com 36,7. Observa-se, a partir desses dados, que apesar da mastectomia total ser, entre as mastectomias, a que menos deixa sequela na mulher, ela ainda é traumatizante pela própria imagem corporal alterada. Acredita-se também que esse achado pode ter sido pelo fato de a amostra ter predominado em $47,1 \%$ de mulheres com mastectomia total, não sendo, portanto, uma amostra homogênea, e pela média de idade das mulheres dessa modalidade cirúrgica ter sido de 56 anos, sendo essa a média de idade das mulheres que obtiveram o pior escore de nulo a ruim.

Quanto aos resultados do QS-F relativos ao desempenho e à satisfação sexual, a maioria das mulheres $(35,3 \%)$ analisadas na atual pesquisa foi classificada na categoria de nulo a ruim, 29,4\% como desfavorável a regular e poucas atingiram o escore classificado de bom a excelente e regular a bom, com 5,9\% em ambos, o que difere de um estudo $^{(20)}$ que utilizou o QS-F com 42 mastectomizadas e encontrou apenas $7,1 \%$ com padrão de nulo a ruim e $40,5 \%$ de desfavorável a regular. Em outra pesquisa ${ }^{(26)}$, com 11 pacientes mastectomizadas, 36,36\% apresentaram desempenho desfavorável a regular; $45,46 \%$, de regular a bom; e nenhuma apresentou a pior categoria. Essa diferença pode dar-se devido a um dos estudos ${ }^{(26)}$ ter sido realizado somente com mulheres casadas e que estavam em tratamento fisioterápico em uma clínica-escola, assim como no trabalho ${ }^{(20)}$ em que a coleta de dados foi realizada em pacientes que estavam em reabilitação em um hospital de grande porte, podendo já existir uma melhor aceitação do seu corpo e uma diminuição das sequelas devido à reabilitação.

A literatura confirma a alta prevalência de disfunções sexuais em mulheres mastectomizadas, variando de 50 a $90 \%(20,22,24,27,28)$, corroborando com o achado da presente pesquisa, na qual $88,2 \%$ das mulheres tinham ao menos uma disfunção sexual, sendo que das 34 mulheres, 88,2\% apresentaram dificuldade na excitação, $82,4 \%$ desejo sexual hipoativo, e 79,4\% referiram dispareunia e anorgasmia.

A disfunção sexual pode vir concomitante às alterações fisiológicas e cirúrgicas e na autoimagem, assim como no uso de medicamentos ou tratamentos que inibem a libido ou que causam secura e atrofia vaginal, sendo que esses fatores podem coexistir com o curso da doença e no póstratamento, podendo ser essa a razão das disfunções sexuais serem tão frequentes em pacientes oncológicos ${ }^{(5,6,29)}$.

Como limitações do atual estudo, têm-se a classe social das entrevistadas e o tipo de cirurgia realizada, pois eram homogêneas, classes sociais desprivilegiadas e mastectomia total, respectivamente, o que pode levar a um pior prognóstico e níveis de QS-F. Esses resultados demonstram o quanto a mulher mastectomizada se retrai para a atividade sexual, fazendo com que o sexo seja desagradável por conta da sua autoimagem e estima, que sofrem alterações devido ao tratamento não conservador. Sugere-se que sejam realizados novos estudos nesse aspecto, através de uma amostra maior, com o intuito de demonstrar que a sexualidade da mulher mastectomizada sofre impacto significativo, a fim de promover um amplo conhecimento das consequências dos procedimentos e doenças nestas, melhorando os objetivos das políticas públicas e a abordagem do profissional de saúde.

\section{CONCLUSÃO}

De acordo com os resultados encontrados no estudo, observa-se que a maioria das mulheres mastectomizadas investigadas mostra função sexual baixa e insatisfatória de acordo com o QS-F, eram casadas e apresentavam até o ensino fundamental.

\section{REFERÊNCIAS}

1. Ministério da Saúde(BR), Instituto Nacional do Câncer. Estimativa de câncer: incidência de câncer no Brasil. [Internet]; 2014 [acesso em 2014 jan 06]. Disponível em: http://www.inca.gov.br/estimativa/2014/sintesede-resultados-comentarios.asp 
2. Ministério da Saúde (BR), Instituto Nacional do Câncer. Controle do Câncer de Mama: documento de consenso. [Internet]; 2004 [acesso em 2014 jan 07]. Disponível em: http://www.inca.gov.br/publicacoes/ consensointegra.pdf

3. Santos D, Vieira E. Imagem corporal de mulheres com câncer de mama: uma revisão sistemática da literatura. Ciênc Saúde Coletiva. 2011;16(5):2511-22.

4. Hughet P, Morais S, Osis M, Pinto Neto A, Gurgel M. Qualidade de vida e sexualidade de mulheres tratadas de câncer de mama. Rev Bras Ginecol Obstet. 2009;31(2):61-7.

5. Krychman ML, Katz A. Breast Cancer and sexuality: multi-modal treatment options. J Sex Med. 2012;9(1):513.

6. Fleury HJ, Pantaroto HSC, Abdo CHN. Sexualidade em Oncologia. Diagn Tratamento. 2011;16(2):86-90.

7. Rohden F. Diferenças de gênero e medicalização da sexualidade na criação do diagnóstico das disfunções sexuais. Estud Feministas. 2009;17(1):89-109.

8. Abdo CHN. Quociente sexual feminino: um questionário brasileiro para avaliar a atividade sexual da mulher. Diagn Tratamento. 2009;14(2):89-1.

9. Abdo CHN. Elaboração e validação do quociente sexual - versão feminina, uma escala para avaliar a função sexual da mulher. Rev Bras Med. 2006;63(9):477-82.

10. Thiel RRC, Dambros M, Palma PCR, Thiel M, Riccetto CLZ, Ramos MF. Tradução para português, adaptação cultural e validação do Female Sexual Function Index. Rev Bras Ginecol Obstet. 2008;30(10):504-10.

11. Souto MD, Souza IEO. Sexualidade da mulher após a mastectomia. Esc Anna Nery Rev Enferm 2004;8(3):402-10.

12. Cassali GD, Gomes NF, Paim C, Amaral CM. Assistência Fisioterapêutica a Pacientes pós- cirurgia do Câncer de Mama. In: $2^{\circ}$ Congresso Brasileiro de Extensão Universitária; 2004. Belo Horizonte. Anais. Belo Horizonte; 2004.

13. Ferreira PCA, Neves NM, Correa RD, Barbosa SD, Paim C, Gomes NF, et al. Educação e assistência fisioterapêutica às pacientes pós-cirurgia do câncer de mama. In: $8^{\circ}$ Encontro de Extensão da UFMG; 2005. Belo Horizonte. Anais. Belo Horizonte; 2005.

14. Talhaferro B, Lemos SS, Oliveira E. Mastectomia e suas conseqüências na vida da mulher. Arq Ciênc Saúde. 2007;14(1):17-22.
15. Associação Brasileira de Empresas de Pesquisa. Critério de Classificação Econômica Brasil 2008 [Internet]. ABEP - Associação Brasileira de Empresas de Pesquisa; 2010 [acesso 2013 maio 15]. Disponível em: http://www.abep.org/new/criterioBrasil.aspx

16. Alicikus ZA, Gorken IB, Sen RC, Kentli S, Kinai $\mathrm{M}$, Alanyali $\mathrm{H}$, et al. Psychosexual and body image aspects of quality of life in Turkish breast cancer patients: a comparison of breast conserving treatment and mastectomy. Tumori. 2009;95(2):212-8.

17. Maluf MFM. O perfil da sexualidade em mulheres com câncer de mama [dissertação]. São Paulo: Universidade de São Paulo; 2008.

18. Moreira JR, Sabino Neto M, Pereira JB, Biasi T, Garcia EB, Ferreira LM. Sexualidade de mulheres mastectomizadas e submetidas à reconstrução mamária. Rev Bras Mastologia. 2010;20(4):177-182.

19. Majewski JM, Lopes ADF, Davoglio T, Leite JCC. Qualidade de vida em mulheres submetidas à mastectomia comparada com aquelas que se submeteram à cirurgia conservadora: uma revisão de literatura. Ciênc Saúde Coletiva. 2012;17(3):707-16.

20. Manganiello A. Sexualidade e qualidade de vida da mulher submetida à mastectomia [dissertação]. São Paulo: Universidade de São Paulo, 2008.

21. Menke CH, Biazús JV, Xavier NL, Cavalheiro JA, Rabin EG, Bittelbrunn A, et al. Rotinas em Mastologia. $2^{\mathrm{a}}$ ed. Porto Alegre: Artmed, 2007.

22. Panjari M, Bell RJ, Davis SR. Sexual Function after Breast Cancer. J Sex Med. 2011;8(1):294-302.

23. Yang EJ, Kim SW, Heo CY, Lim JY. Longitudinal changes in sexual problems related to cancer treatment in Korean breast cancer survivors: a prospective cohort study. Support Care Cancer. 2011;19(7):909-18.

24. Sbitti Y, Kadiri H, Essaidi I, Fadoukhair Z, Kharmoun $\mathrm{S}$, Slimani K, et al. Breast cancer treatment and sexual dysfunction: Moroccan women's perception. BMC Women's Health. 2011;11(29):1-5.

25. Backwell RT, Volker DL. Sexual dysfunctions related to the treatment of young women with breast cancer. Clin J Oncol Nurs. 2005;9(6):697-701.

26. Correia GN, Oliveira J, Mesquita-Ferrari RA. Avaliação da qualidade de vida em mulheres submetidas à mastectomia radical e segmentar. Fisioter Pesqui. 2007;14(3):31-6.

27. Carvalho DM, Martins C, Rezende LF. Sexualidade no pós-operatório de câncer de mama. Rev Pensamento Plural. 2011;5(2):60-4. 
28. Gahm J, Wickman M, Brandberg Y. Bilateral prophylactic mastectomy in women with inherited risk of breast cancer--prevalence of pain and discomfort, impact on sexuality, quality of life and feelings of regret two years after surgery. Breast. 2010;19(6):4629.

29. Emilee G, Ussher JM, Perz J. Sexuality after breast cancer: a review. Maturitas. 2010;66(4):397-407.

\section{Endereço de correspondência:}

Izabelle Quintiliano Montenegro Bomfim

Universidade Estadual de Ciências da Saúde de Alagoas

Rua Doutor Jorge de Lima, 113

Bairro: Trapiche da Barra

CEP: 57010-300 - Maceió - AL - Brasil

E-mail: izabellebomfim@hotmail.com 